\title{
Research on the real-time business intelligence framework of ROA mobile
}

\author{
Zhu Zebo ${ }^{1}$, Chen Jian $^{1}$ \\ 1.China Satellite Maritime Tracking and Controlling Department, Jiangyin 214431, China
}

\begin{abstract}
Keyword: Business intelligence, mobile real-time business intelligence, Android oriented architecture resources
\end{abstract}

\begin{abstract}
The emergence of enterprise business intelligence system can not solve the traditional database massive data intelligent analysis and forecasting problems. With intelligent, enterprises and organizations popularity and mobile communications equipment worldwide mobile communications needs of mobile business has gradually increased, so that the mobile business intelligence has become possible. As traditional business intelligence hardware and software infrastructure requirements may not be fully realized in the current class mobile terminals, for business intelligence applications present situation, proposed architecture is based on ROA mobile real-time business intelligence systems, the architecture of the server architecture, mobile client architecture and communication security mechanisms in detail, and the use of Android architecture by examples of the key technologies to achieve a detailed exposition.
\end{abstract}

\section{Introduction}

With the accumulation of corporate information resources, more and more companies hope to multi-dimensional business data analysis and mining, provide a reliable basis for decision making. Business Intelligence (Business Intelligence, BI) is a data warehouse made (or data mart), query reporting, data analysis and data mining and other components, and can support real-time business decision-making and business management activities and timely adapt to the market An application of technology needs ${ }^{[1]}$.

The obvious shortcomings of traditional BI and integration of real-time presence in other aspects of data and information, companies often only local query analysis, it is often difficult to timely and accurate understanding of the internal information and external market dynamics, specific performance: 1 ) BI lack of real-time, can not effectively solve the data lag, analysis and decision-making lag lag; 2) BI system features a single, lack of standardization and integration. Most business units carry out a sectoral business intelligence applications, intersectoral due their business intelligence products with unique user paradigm, a proprietary format, and metadata management, so that the analysis results are inconsistent, information sharing difficult.

In recent years, domestic and foreign scholars have done a lot of business intelligence in real-time in-depth research. Richard Hackathon presented in real-time business intelligence direction of a value model, pointing out the value of BI will be lost in the event and the response from the corporate segment and presents the underlying data (Data Latency), potential analysis (Analysis Latency), potential Decision (Decision Latency) three concept [2], it is because of the value of these three will be loss making companies miss potential opportunities. Zhou Jin for real-time business intelligence business decisions were related research, and knowledge management (KM) and business intelligence relationship depth analysis, the analysis can handle enterprise data, information, knowledge, support business activities Integration Model between knowledge and business intelligence both the KMBI [3]. Shao Liang, who proposed a real-time business intelligence framework, and with existing enterprise applications (such as SCM, CRM, KM) and other integration and enables a seamless connectivity [4] between Business Intelligence and Enterprise Applications. Thus, real-time business intelligence and integration has been considered one of the future research focus and application trends in the field.

Mobile BI business intelligence research in the field of a new subject, which by means of mobile 
communication technologies and means, the real-time BI and integration into one. In this paper, business intelligence and application status of smart mobile devices, through the traditional business intelligence systems architecture to transform the proposed mobile real-time business intelligence (Mobile Real-Time Business Intelligence, MRBI based on ROA is based on ROA mobile real-time business intelligence to effectively solve BI data transmission lag analysis lag.

\section{The ROA Mobile real-time business intelligence framework}

ROA (Resource-Oriented Architecture, resource-oriented architecture) that contains the client server, stateless, cache, resources uniqueness, a layered architecture and scalability and on-demand to provide code six constraints ${ }^{[5]}$. And traditional SOA (Service-Oriented Architecture, service-oriented architecture) WEB service, compared with the advantages of WEB services Based on ROA in scalability, security, data coupling, etc., can effectively reduce system development complexity, improve system portability ${ }^{[6]}$. MRBI client uses the time polling mechanism when in fact increase.

\subsection{MRBI server architecture}

BI systems without affecting the original application on the basis of which it is possible to provide services modules packaged as REST resources, which will move existing BI and enterprise BI systems for seamless system integration. MRBI system integration architecture shown in Figure 1.

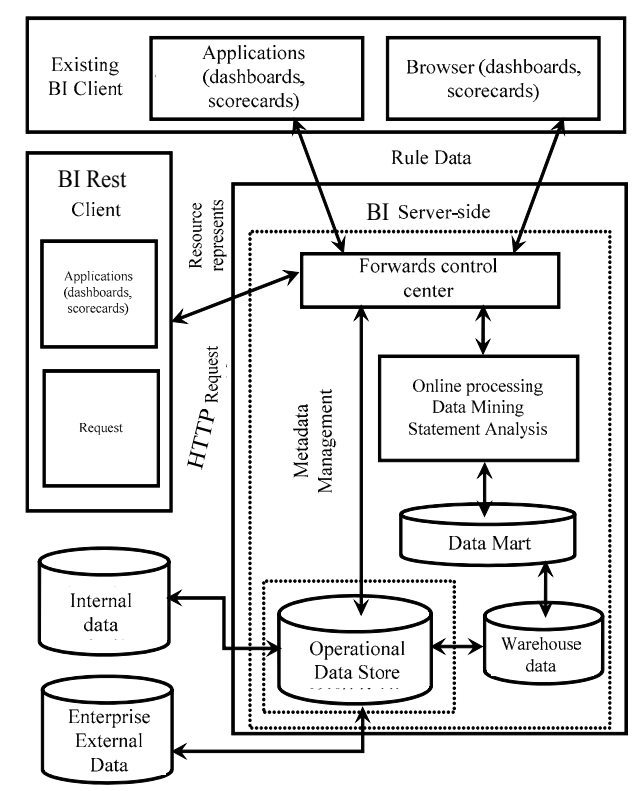

Figure 1BI systems integration architecture diagram

Real-time business intelligence system server resolve data ETL (extract, transform, load) and real-time data analysis. In Figure 1, the internal and external data through ETL operations into corporate decision-making, operation and management of useful information or knowledge to metadata management in data storage warehouse. Operational data store is mainly used to store various types of data generated in the production and operation activities, it only holds the current data, and automatically added to the data warehouse on a regular basis in order to meet the needs of real-time BI systems. Real-time requirements for higher BI data can be published directly through metadata management to the mobile client.

The server can be a data warehouse or data warehouse, data marts data mining, online analytical processing technology processing, so that data and information into knowledge of decision support, knowledge in an appropriate manner (such as dashboards, scorecards) show In the user view.

BI server accepts client requests, which control the forwarding center on the basis of the request (eg REST HTTP PUT, GET requests) and request URI characteristics resolution request to the appropriate business needs. BI system based on business needs to read from data marts, operational data in the database or directly read real-time data, through data analysis (online analytical 
processing, data mining, reporting, analysis, etc.) are converted into rules data or resources represented by the control Forwarding Centre transferred to BI clients.

\subsection{Architecture MRBI mobile terminal system}

Based on the server architecture of the BI system, Figure 2 shows the architecture of the system Based on ROA MRBI system for mobile terminals. Mobile client uses the time polling mechanism timely access to BI data to achieve real-time data refresh knowledge. In order to speed up the process or reduce network data transmission, can be important information by certain metadata management kept safe in a mobile terminal. Data and information of the mobile terminal can only read from the enterprise data warehouse server (or database), or from other mobile terminal system knowledge data stored in the database.

MRBI mobile client system-critical processes are as follows: 1) mobile intelligent terminal itself, the data management system (such as Android contact management systems) and BI systems to extract, transform, load the data source, the resulting data is stored in the mobile terminal data warehouse and BI systems database ; and 2) the mobile terminal sends REST network requests to obtain data (such as reports, key performance indicators, etc.) sent by the server; 3) BI system based on business needs, the received data is stored in the underlying data warehouse or data received directly data analysis layer; 4) operational data store analysis results obtained by analyzing the data, and based on business requirements will result data stored in the data warehouse BI; 5) the use of mobile intelligent terminal system will display the data in different ways in the user view in.

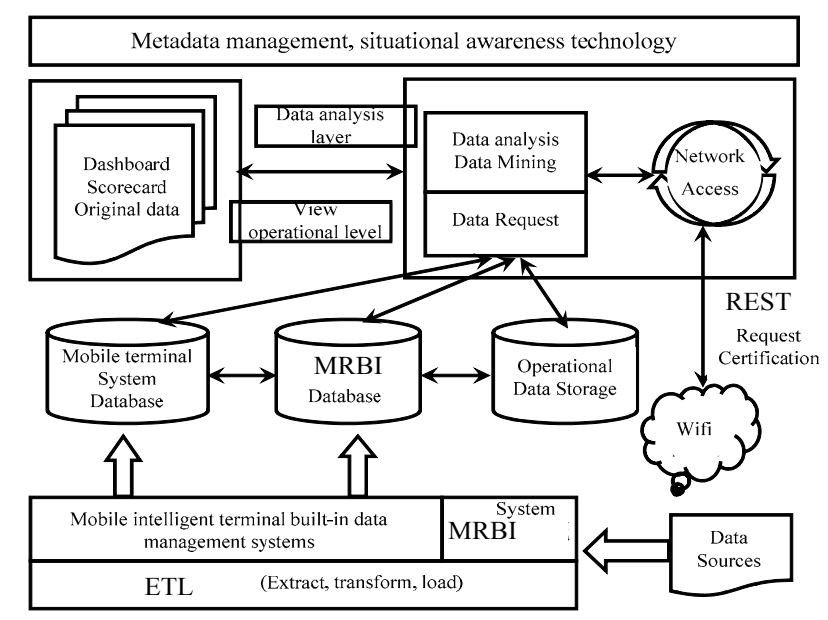

Figure 2 Mobile terminal business intelligence processing logic processing architecture

\subsection{MRBI mobile end-user access authentication and communication security}

Set incorrectly or the threat of unauthorized operation and network monitoring to intercept wireless data network communications permissions, presented ROA wireless network access and secure communication framework is based, as shown in Figure 3.

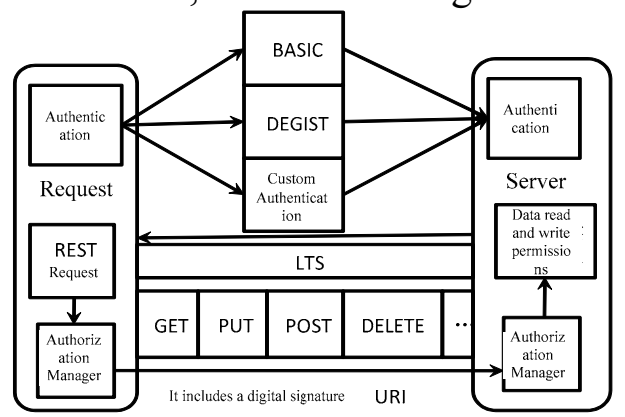

Figure 3 MRBI system for mobile terminal access authentication and secure communication In the following ways to enhance the protection of data security and resources:

1) client authentication, ensure that the user legitimacy. Depending on usage, you can choose 
HTTP BASIC, DIGEST or custom access authentication (such as Amazon's Simple Storage Service);

2) For user authorization and access control using expressly purview. Assign different privileges to different users, to avoid unauthorized tampering with the data.

3 ) the use of a specific algorithm URI of digital signatures (eg HMAC-SHA1 and RSA-SHA1 algorithm) data, the signature as a query parameter is included in the resource the URI included in the URI to protect sensitive data integrity or confidentiality .

4) Use TLS (Transport Layer Security, Transport Layer Security), while the preparation of the server, only allow HTTPS requests to access confidential data resources, so as to maintain the confidentiality and security of the resource data.

\section{Achieve MRBI Architecture}

Based on the basis of the logical structure of the above-mentioned server and mobile terminals on, here is an example of realization of MRBI ROABased on system. In this example, the server uses JAVA WEB application and RESTLET integration mode. Phone mobile terminals using Android system. The main flow MRBI system implementation and some of the code shown in Figure 4.

1) RESTLET and WEB application integration. Spring provides an integrated interface RESTLET integrated JAVA WEB applications.

2) The client uses REST HTTP network request.

3) resolve REST server requests from clients and perform data analysis. Server based REST requests for data analysis and the results of data encapsulated in resource transfer to the client.

4) The client accepts return data will be returned data storage, analysis and presentation.

\begin{tabular}{|c|c|}
\hline $\begin{array}{ll} & 1 \\
\text { RESTLET } \backslash \text { WEB Application } \\
\end{array}$ & $\begin{array}{l}2 \text { Mobile terminal analysis and } \\
\text { presentation }\end{array}$ \\
\hline $\begin{array}{l}\text { <servlet }> \\
<\text { servlet-name>restlet</servlet-name> } \\
\text { <servlet-class> } \\
\text { com.noelios.restlet.ext.spring.SpringServerServlet } \\
</ \text { servlet-class }> \\
\text { <init-param }> \\
<\text { param-name>org.restlet.component</param- } \\
\text { name> } \\
<\text { param-value>component</param-value }> \\
</ \text { init-param }>\text {... }\end{array}$ & $\begin{array}{l}\text { // Send Intent } \\
\text { return } \\
\text { ChartFactory. } \\
\text { getCubicLineChartIntent } \\
\text { (context, } \\
\text { buildBarDataset(titles, values), } \\
\text { renderer, } 0.5 f \text { ); }\end{array}$ \\
\hline 2 Service from the client-side parsing REST & $\begin{array}{ccc}\begin{array}{c}\text { Mobile } \\
\text { terminal }\end{array} & 3 & \text { REST HTTP } \\
\text { Network } \\
\text { requests }\end{array}$ \\
\hline $\begin{array}{l}\text { PUT acceptRepresentation(Representation entity)\{...\} } \\
\text { GET getRepresentation(Variant variant) }\{. . .\} \\
\text { DELETE } \quad \text { removeRepresentations }()\{\ldots\} \\
\text { POAST storeRepresentation(Representation entity)\{...\} }\end{array}$ & $\begin{array}{l}\text { resource } \\
\text { ClientResource Classes instances } \\
\text { rep Representation } \\
\text { resource .delete(); } \\
\text { resource post(rep); } \\
\text { resource.put(rep); } \\
\text { resource.get(); }\end{array}$ \\
\hline
\end{tabular}

Figure 4 MRBI systems and processes to achieve part of the code

Below 2010 - 2011 annual sales of a business analysis, for example, mobile client accepts BI data, use Android widget achartengine graphic plotting. 2010 sales compared with 2011 page views Figure 5 is a mobile terminal (mobile phone) display a line graph form multi-dimensional analysis of the data. 


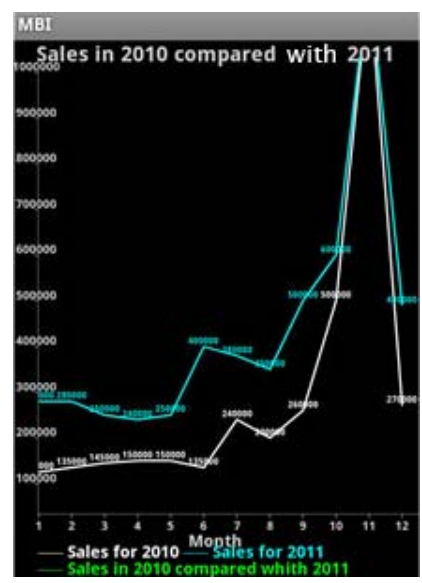

Figure 5 Realization of Android phones business intelligence system example of FIG.

\section{Conclusion}

Traditional business intelligence systems have been developed to face greater challenges. With innovative knowledge management, data analysis and mining technology, mobile communication technology, real-time business intelligence, mobile business intelligence, business intelligence, next-generation cloud computing technology will become mainstream enterprise BI applications. This paper presents and analyzes the mobile real-time business intelligence systems for the mobile environment is change traditional business intelligence systems and improvements. The importance of corporate or organizational business intelligence system between the mobile terminal meta data management and data security storage for further study and discussion. HTTP implement custom authentication systems to improve data transmission security in-depth study and implementation pending.

\section{References}

[1]Hugh J.Watson .Tutorial: Business Intelligence -Past, Present, and Future [J]. Communications of the Association for Information Systems, 2009.

[2] Richard Hackthorn The BI Watch:. Real-Time to Real Value [J] .DM Review, 2004, 14 (1): 24, 43.

[3] Leonard Ricbardson, Sam Ruby of. Landsat, Li Hongjun, Hu Wei translation. Restful Web Services [M]. 1st edition. Beijing Electronic Industry Press .2008.

[4] Subbu Allamaraju with. Ding Xuefeng, often translated. RESTful Web Services Cookbook [M]. 1st edition. Beijing Electronics Industry Press .2011.

[5] Feng Xinyang, Shen Jian Beijing R ES T and RP C:. Comparative analysis of two architectural styles We b Services [J] Computer Systems, 2010, 31 (7): 1393-1395. 